Supporting Information

\title{
DNA-Assisted Exfoliation of Tungsten Dichalcogenides and Their Antibacterial Effect
}

Gyeong Sook Bang, ${ }^{\dagger}, \#$ Suhyung Cho, ${ }^{\ddagger *}$ Narae Son, ${ }^{\dagger}$ Gi Woong Shim, ${ }^{\dagger}$ Byung-Kwan Cho, ${ }^{\ddagger}$ and Sung-Yool Choi $*{ }^{\dagger}$

${ }^{\dagger}$ School of Electrical Engineering, Graphene Research Center, Korea Advanced Institute of Science and Technology (KAIST), Daejeon 34141, Republic of Korea

${ }^{\ddagger}$ Department of Biological Science and KI for the BioCentury, Korea Advanced Institute of Science and Technology (KAIST), Daejeon 34141, Republic of Korea

* Corresponding Author

Tel. +82 42350 7427. Fax: +82 42350 7283. E-mail: sungyool.choi@kaist.ac.kr 
We first had conducted preliminary test for $\mathrm{MoS}_{2}$ exfoliation using commercial DNA (SigmaAldrich, salmon testes) of low (short-DNA, approximately 200 base pairs) and high (longDNA, 2000 base pairs) molecular weight under same condition. But the exfoliation yield using low molecular-weight ssDNA was very low of $<10 \%$, which was lower exfoliation efficiency than the reported data $(\sim 10 \%)$ for aqueous $\mathrm{MoS}_{2}$ exfoliation using sodium cholate. The exfoliation yield did not improve even under conditions of excess short-DNA of over 20 times and lengthy sonication time $(\sim 8 \mathrm{~h})$. On the contrary, the high molecular-weight ssDNA showed high yield of more than $80 \%$. Thus, for high exfoliation yield of $\mathrm{WX}_{2}$, we used ssDNA of the high molecular weight ( 2000 base pairs, $42 \%$ G-C).

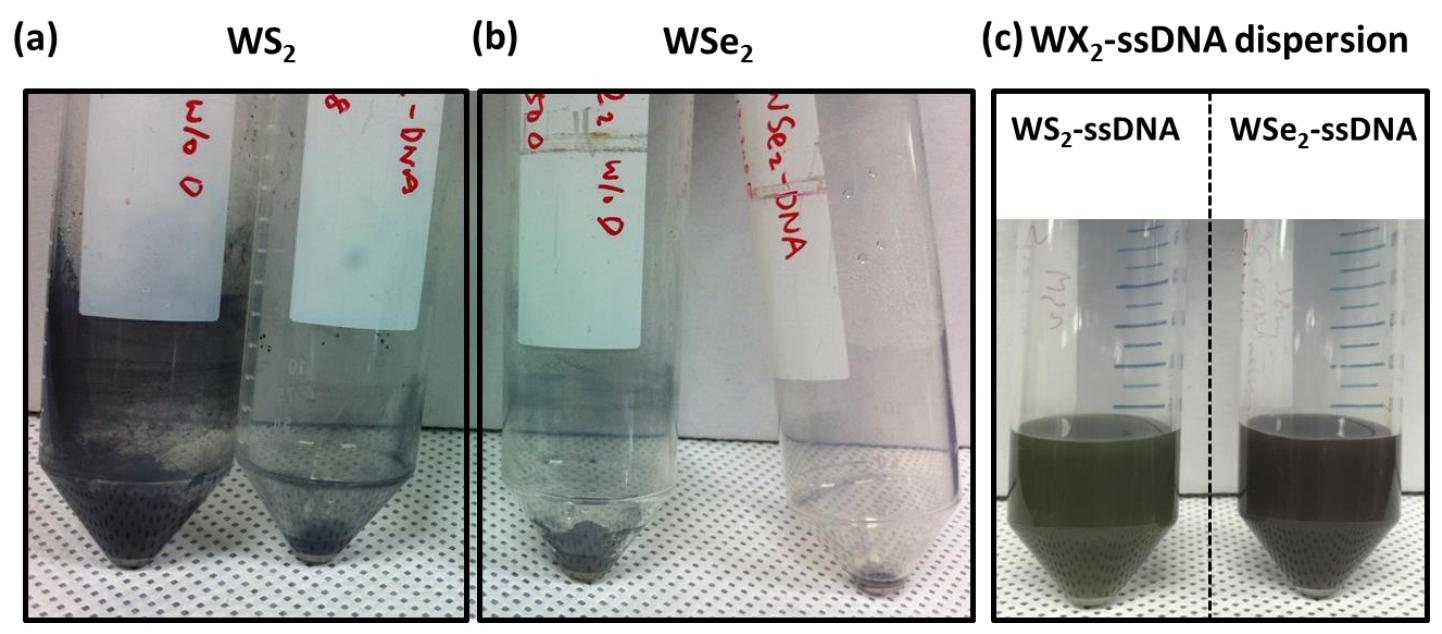

Figure S1. ( $\mathrm{a}, \mathrm{b})$ Photographs of the sediment of $\mathrm{WX}_{2}$ treated in the absence (left tube) and in the presence of (right tube) ssDNA: (a) $\mathrm{WS}_{2}$, (b) $\mathrm{WSe}_{2}$. (c) Photographs of $\mathrm{WS}_{2}$-ssDNA and $\mathrm{WSe}_{2}$-ssDNA dispersion. 


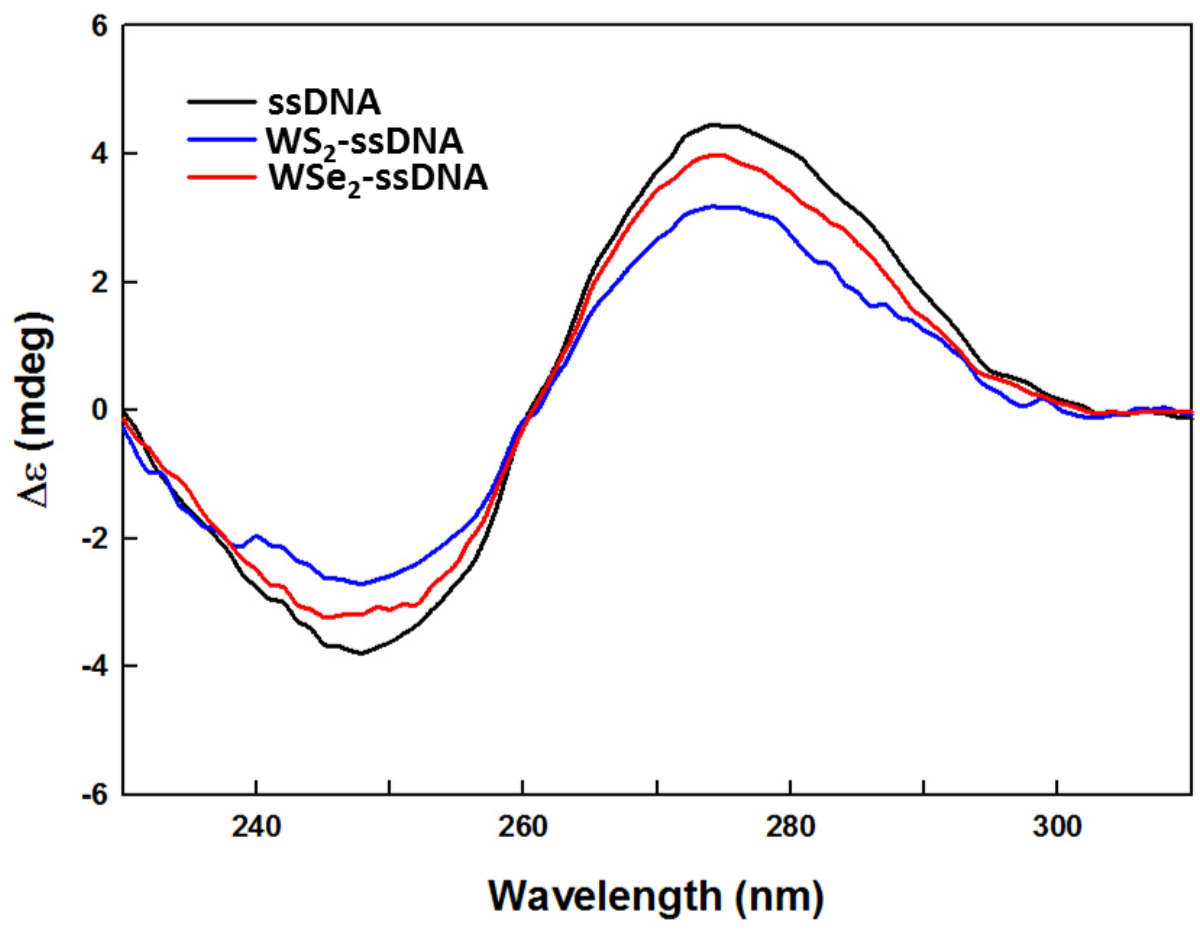

Figure S2. CD spectra for ssDNA (black line), $\mathrm{WS}_{2}$-ssDNA (blue line), and $\mathrm{WSe}_{2}$-SsDNA (red line) dispersion. 
(a)

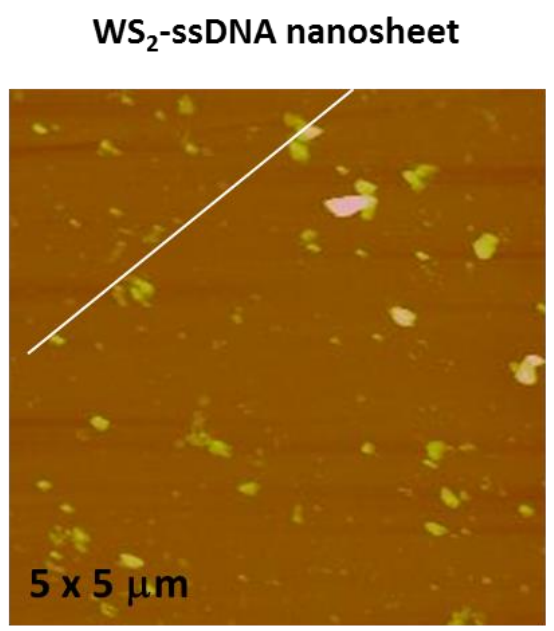

(b)

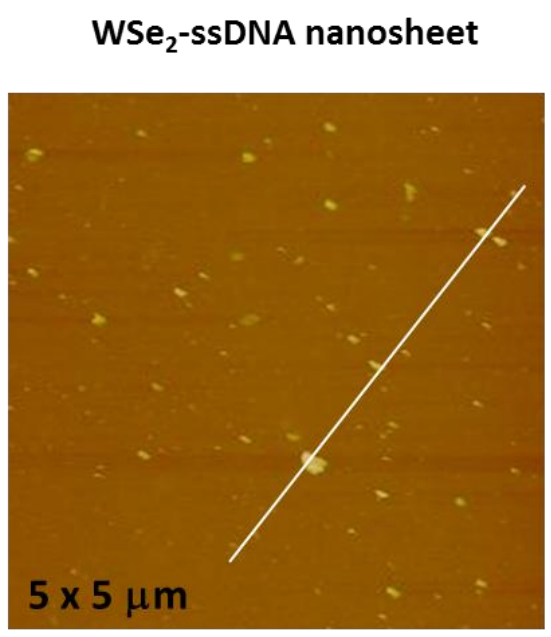

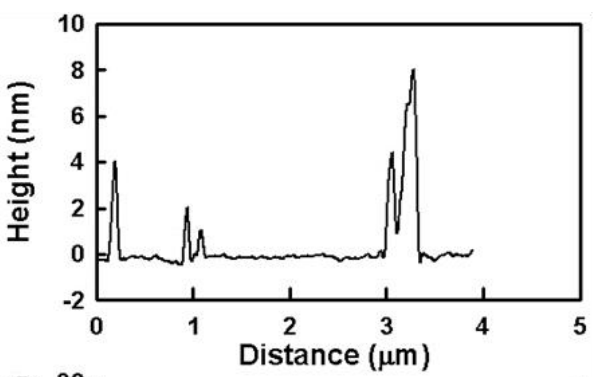
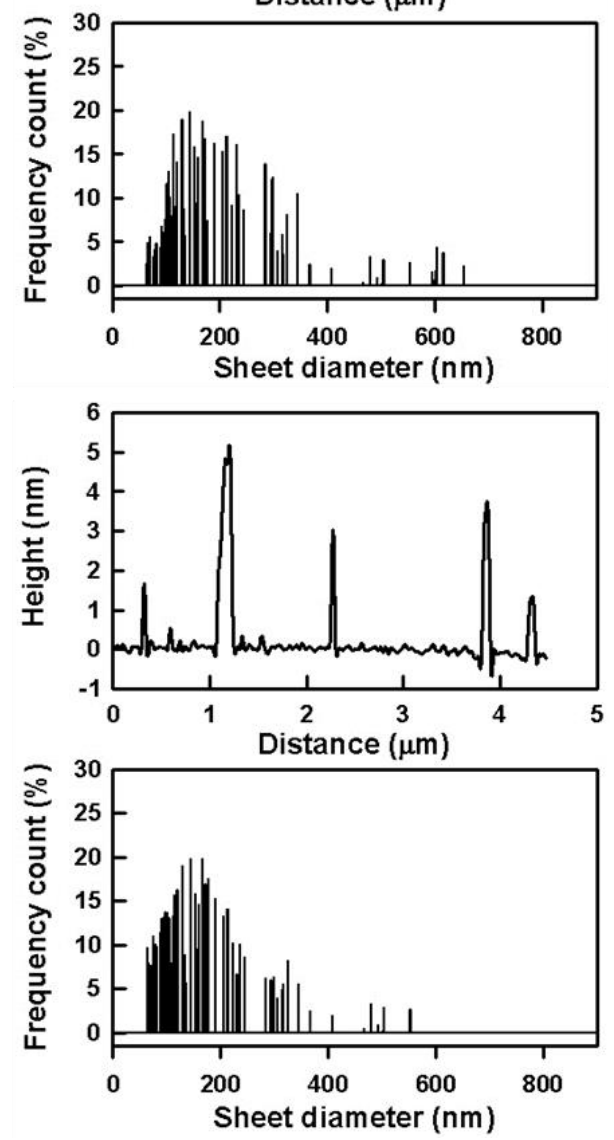

Figure S3. Tapping-mode AFM images (left), corresponding line scans (right upper) and size distribution of sheet diameter (right down) for $\mathrm{WS}_{2}$-ssDNA (a) and $\mathrm{WSe}_{2}$-ssDNA (b) nanosheets on a $\mathrm{SiO}_{2} / \mathrm{Si}$ substrate. 
(b)

(a)
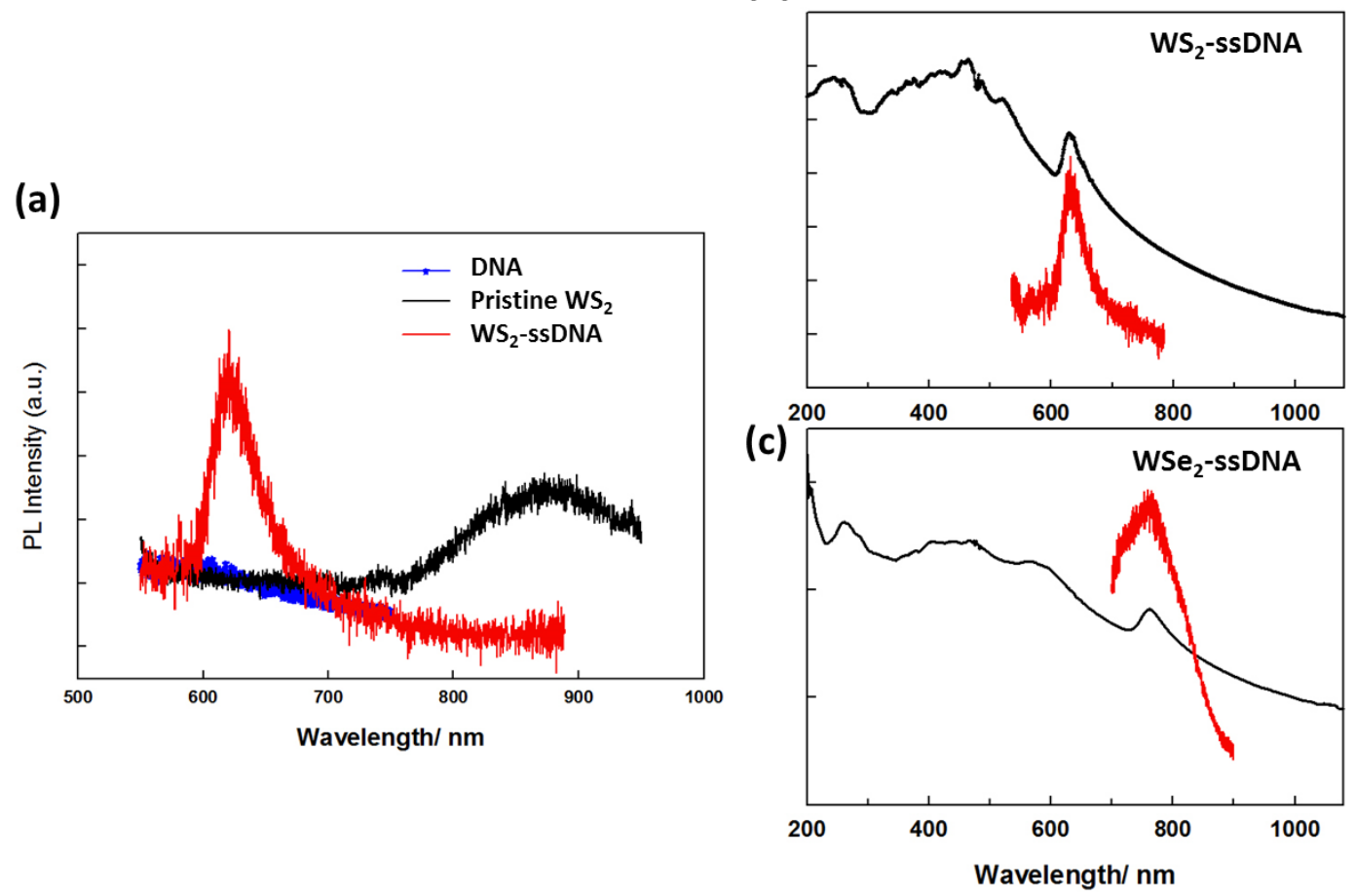

Figure S4. (a) PL spectra for ssDNA (blue line), pristine $\mathrm{WS}_{2}$ powder (black line), and $\mathrm{WS}_{2}$ ssDNA nanosheets (red line) on a $\mathrm{SiO}_{2} / \mathrm{Si}$ substrate. (b, c) UV-Vis (black line) and PL (red line) spectra of $\mathrm{WX}_{2}$-ssDNA nanosheet: (b) $\mathrm{WS}_{2}$-ssDNA and (c) $\mathrm{WSe}_{2}$-ssDNA.

(a)

(b)
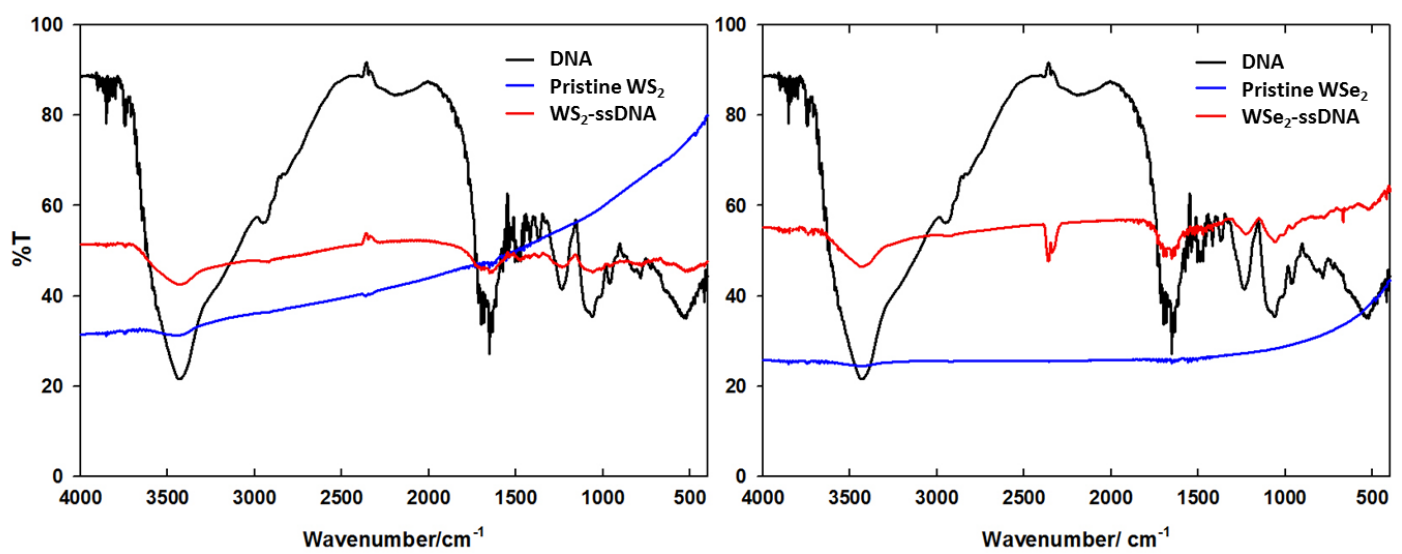

Figure S5. FT-IR spectra for DNA (black line), pristine $\mathrm{WX}_{2}$ powder (blue line), and $\mathrm{WX}_{2^{-}}$ ssDNA nanosheets (red line): (a) $\mathrm{WS}_{2}$ and (b) $\mathrm{Wse}_{2}$. 
(a)

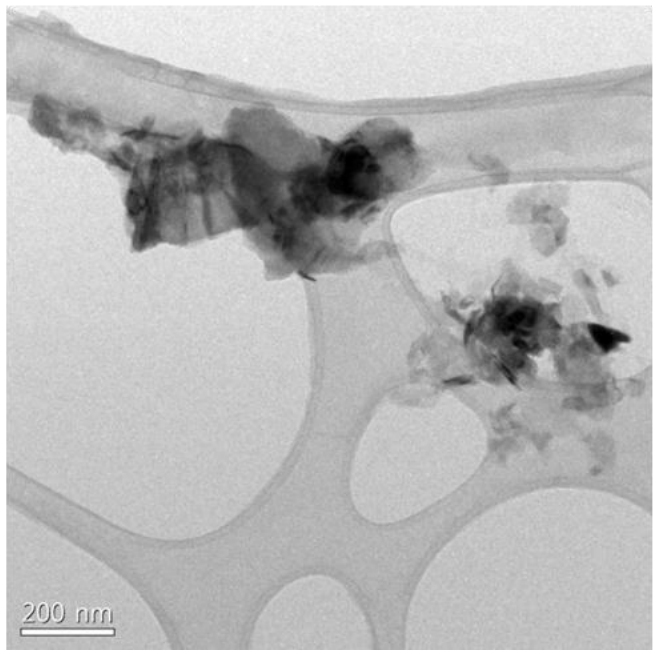

(b)

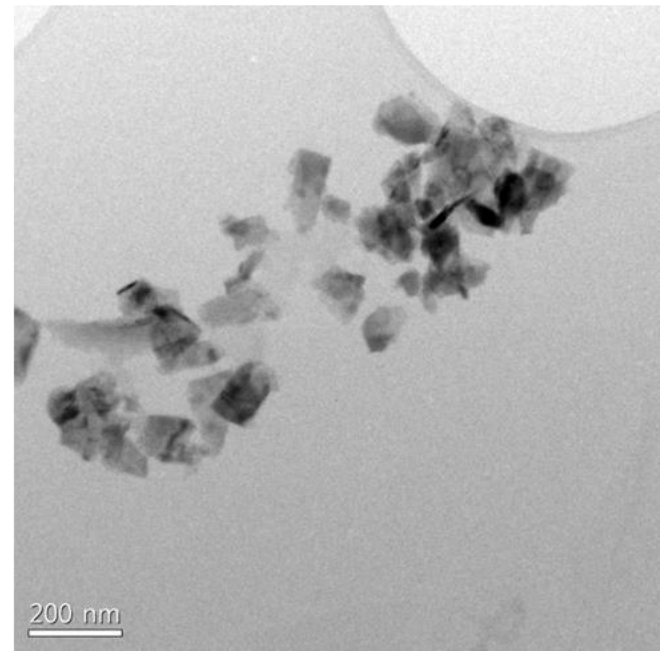

Figure S6. TE micrographs of exfoliated $\mathrm{WS}_{2}$-ssDNA (a) and $\mathrm{WSe}_{2}$-ssDNA (b) nanosheets.

(a)

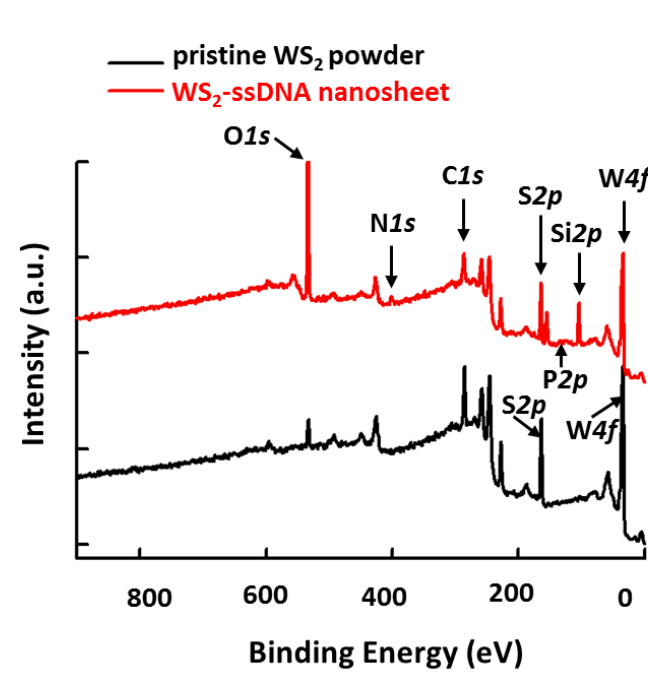

(b)

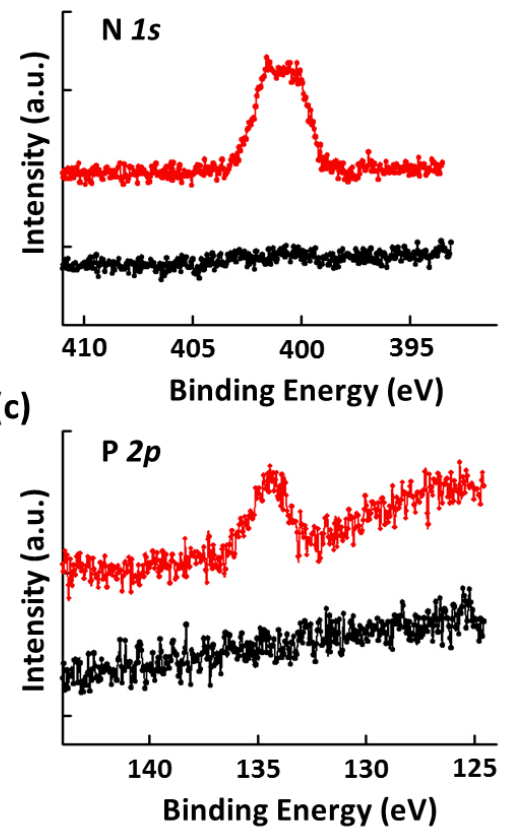

Figure S7. XPS spectra of pristine $\mathrm{WS}_{2}$ powder on carbon tape (black line) and $\mathrm{WS}_{2}$-ssDNA nanosheets on $\mathrm{SiO}_{2} / \mathrm{Si}$ substrate (red line): survey (a) and high resolution of $\mathrm{N} 1 \mathrm{~s}$ and (c) P $2 \mathrm{p}$ (b). 